\title{
Optical transferences and their application to ray tracing through the human cornea*
}

\author{
SD Mathebula ${ }^{\dagger}$ and A Rubin ${ }^{\dagger \dagger}$ \\ †Department of Optometry, University of Limpopo, Private Bag x1106, Sovenga, 0727 South Africa \\ ${ }^{\dagger}$ Department of Optometry, University of Johannesburg, PO Box 524, Auckland Park, 2006 South Africa
}

Received 21 April 2011; revised version accepted 24 October 2011

\begin{abstract}
The purpose of this paper is two fold, firstly to describe aspects of the quantitative analysis of the linear optical character of the corneas of ten young and healthy subjects using the exp-mean-log-transference and secondly to illustrate how mean transference and ray vector fields or diagrams can be used to explain and understand the optical properties of corneas as thick optical systems.

An Oculus Pentacam was used to obtain 43 successive measurements of the radii of curvature of the anterior and posterior corneal surfaces and the central corneal thicknesses of the right eyes of ten subjects. From these measurements $4 \times 4$ ray transferences were calculated. Mean transferences were obtained via multi-dimensional Hamiltonian space and these mean transferences were used to produce stereo-pairs of ray vector fields. The mean trans-
\end{abstract}

ferences are also important in understanding the behaviour of light through each of the corneas concerned.

This paper provides the first order optical characters of corneas from the positions and inclinations of rays entering and leaving such systems. As anticipated, light rays through the cornea are deflected inwards when the refractive index of the cornea is greater than the index of the surrounding medium. The exp-mean-log transference for a specific cornea exists and is the optical transference of the averaged cornea of the sample of measurements for that cornea. Within the limitations of linear or paraxial optics, the corneas of the different eyes in this sample and their averages were found to be close to that of thin optical systems; but they were not truly thin and instead should be considered as being thick optical systems. (S Afr Optom 2011 70(4) 156-167)

\section{Introduction}

The complete first order characterization of an optical system, including an eye or part of an eye, requires knowledge of what the system does to any ray traversing it. In linear optics the optical character of the system about a longitudinal axis is fully represented by the ray transference $\mathbf{T}$ of the system ${ }^{1-4}$. Keating $^{5-7}$ called it the system matrix. The eye or any optical system (the cornea in this paper) operates on the ray according to the general equation ${ }^{4,8-10}$ $\mathbf{T} \gamma_{0}=\gamma$.
The cornea changes the state of the ray (vector) from $\gamma_{0}$ at incidence on the front surface of the cornea to ray vector $\gamma$ at the back surface of the cornea. The emergent ray state is described using a $4 \times 1$ vector with the form

$$
\gamma=\left(\begin{array}{l}
\mathbf{y} \\
\boldsymbol{\alpha}
\end{array}\right)
$$

where $\mathbf{y}$ and $\boldsymbol{\alpha}$ (both $2 \times 1$ ) are the position and reduced inclination at emergence of the ray relative to a longitudinal axis, and similarly for the incident ray state, $\gamma_{0}$. The reduced inclinations are related to the

\footnotetext{
*This paper is based on research for the DPhil degree of the first author with the supervision of Professors A Rubin and WF Harris
} 
(unreduced) inclinations $\mathbf{a}_{0}$ and $\mathbf{a}$ with local indices of refraction $n_{0}$ and $n$ by $\boldsymbol{\alpha}_{0}=n_{0} \mathbf{a}_{0}$ and $\boldsymbol{\alpha}=n \mathbf{a}$. Generally the ray transference $\mathbf{T}$ of an optical system is a $5 \times 5$ matrix $^{8}$ but where tilts and decentration of the optical system are ignored the transference becomes a $4 \times 4$ matrix $^{8-12}$, that is,

$\mathbf{T}=\left(\begin{array}{ll}\mathbf{A} & \mathbf{B} \\ \mathbf{C} & \mathbf{D}\end{array}\right)$.

Here the $2 \times 2$ sub-matrices A (dilation), B (disjugacy), $\mathbf{C}$ (divergence) and $\mathbf{D}$ (divarication) are the fundamental first order optical properties of a centred optical system. There are relationships among these four fundamental properties and other first order optical properties of the system can be derived from these four properties. One of the derived properties is the dioptric power matrix $\mathbf{F}$ of the system defined by ${ }^{1,9}$

$\mathbf{F}=-\mathbf{C}$.

Another derived property is the corneal plane refractive compensation for an eye given by ${ }^{1,9}$

$$
\mathbf{F}_{0}=\mathbf{B}^{-1} \mathbf{A} \text {. }
$$

The transference is symplectic, that is, it has a unit determinant. The 16 components of the $4 \times 4$ transference are not independent and they are related by a set of six equations called the symplectic relations ${ }^{8,13}$. $\mathbf{T}$ is a $4 \times 4$ symplectic matrix ${ }^{1,13}$, that is, it obeys $\mathbf{T}^{\mathrm{T}} \mathbf{E T}=\mathbf{E}$ where

$$
\mathbf{E}=\left(\begin{array}{cc}
\mathbf{O} & \mathbf{I} \\
-\mathbf{I} & \mathbf{O}
\end{array}\right)
$$

and $\mathbf{O}$ and $\mathbf{I}$ are the $2 \times 2$ null and identity matrices.

To determine the transference of the cornea one needs measurements of three elements of the cornea, namely, the central powers of the anterior and posterior corneal surfaces and also the central corneal thickness. In this study the refractive indices of the cornea and the aqueous were taken as 1.376 and 1.336 , respectively. The cornea has an anterior surface of power $\mathbf{F}_{\mathrm{a}}$, reduced thickness $\tau$ and a posterior surface of power $\mathbf{F}_{\mathrm{p}}$. Then the transference of the cornea $\mathbf{T}_{\mathrm{c}}$ is the product, in reverse order, of the three corresponding transferences ${ }^{8,14}$. $\mathbf{T}_{\mathrm{a}}$ is given by

$$
\mathbf{T}_{\mathrm{a}}=\left(\begin{array}{cc}
\mathbf{I} & \mathbf{O} \\
-\mathbf{F}_{\mathrm{a}} & \mathbf{I}
\end{array}\right)
$$

$\mathbf{T}_{\mathrm{p}}$ is given by equation 7 with subscript "a" replaced by "p". $\mathbf{T}_{\tau}$ is given by

$$
\mathbf{T}_{\tau}=\left(\begin{array}{cc}
\mathbf{I} & \tau \mathbf{I} \\
\mathbf{O} & \mathbf{I}
\end{array}\right)
$$

where $\tau$ is the reduced thickness of the cornea. Then the transference of the cornea $\mathbf{T}_{c}$ is the product, in reverse order, of the three corresponding transferences 8,14

$$
\mathbf{T}_{\mathrm{c}}=\mathbf{T}_{\mathrm{p}} \mathbf{T}_{\tau} \mathbf{T}_{\mathrm{a}}
$$

where $\mathbf{T}_{\mathrm{a}}$ is the transference of the anterior corneal surface, $\mathbf{T}_{\tau}$ is the transference of the body of the cornea (reduced thickness) and $\mathbf{T}_{\mathrm{p}}$ that of the posterior corneal surface. Multiplication finally results in

$$
\mathbf{T}_{\mathrm{c}}=\left(\begin{array}{cc}
\mathbf{I}-\tau \mathbf{F}_{\mathrm{a}} & \tau \mathbf{I} \\
-\left(\mathbf{F}_{\mathrm{a}}+\mathbf{F}_{\mathrm{p}}-\mathbf{F}_{\mathrm{p}} \tau \mathbf{F}_{\mathrm{a}}\right) & \mathbf{I}-\tau \mathbf{F}_{\mathrm{p}}
\end{array}\right),
$$

which is the transference of the cornea as a part of an eye. In equation $10 \quad \mathbf{F}_{\mathrm{a}}+\mathbf{F}_{\mathrm{p}}-\mathbf{F}_{\mathrm{p}} \tau \mathbf{F}_{\mathrm{a}}$ is the power of the cornea, usually called the equivalent power of the system ${ }^{9}, 14-17$.

Given a set of ray transferences $\mathbf{T}_{1}, \mathbf{T}_{2}, \ldots, \mathbf{T}_{n}$, the arithmetic mean

$\overline{\mathbf{T}}=\frac{1}{N} \sum_{n=1}^{N} \mathbf{T}_{n}$

may not be a ray transference because of the symplectic condition ${ }^{1}, 13,14,18$.

Transferences themselves are not closed under addition and multiplication by a scalar and, hence, are not amenable to standard quantitative analysis such as calculation of an arithmetic mean. Transferences must first be transformed into log-transferences ${ }^{1,2,14,15,19-22}$. Once transformed they constitute a linear or vector space and quantitative analyses such as calculation of means and variance-covariances can be performed in the transformed transference space. For each transference, $\mathbf{T}$, the transformed transference is defined by $\hat{\mathbf{T}}=\log \mathbf{T}$

and the transformed transference $(\hat{\mathbf{T}})$ can be written in terms of sub-matrices as for equation 3

$$
\hat{\mathbf{T}}=\left(\begin{array}{cc}
\hat{\mathbf{A}} & \hat{\mathbf{B}} \\
\hat{\mathbf{C}} & \hat{\mathbf{D}}
\end{array}\right) \text {. }
$$

The transformed transference, $\hat{\mathbf{T}}$, is a Hamiltonian 
matrix that satisfies $\hat{\mathbf{T}}^{\mathrm{T}} \mathbf{E}=\mathbf{E}^{\mathrm{T}} \hat{\mathbf{T}}$ where $\mathbf{E}^{\mathrm{T}}$ is the transpose of $\mathbf{E}$. Because of the Hamiltonian nature of $\hat{\mathbf{T}}$, the Hamiltonian space in which the calculations are performed is 10 -dimensional ${ }^{14,15,22}$. An alternative form of equation 13 can be obtained by expanding $\hat{\mathbf{T}}$ as ${ }^{21,22}$

$$
\begin{aligned}
& \hat{\mathbf{T}}=\hat{A}_{I} \mathbf{I}+\hat{A}_{J} \mathbf{J}+\hat{A}_{K} \mathbf{K}+\hat{A}_{L} \mathbf{L}+\hat{B}_{I} \mathbf{I}+\hat{B}_{J} \mathbf{J} \\
& +\hat{B}_{K} \mathbf{K}+\hat{C}_{I} \mathbf{I}+\hat{C}_{J} \mathbf{J}+\hat{C}_{K} \mathbf{K}
\end{aligned}
$$

where $\mathbf{I}$ is the $2 \times 2$ identity matrix and $\mathbf{J}=\left(\begin{array}{cc}1 & 0 \\ 0 & -1\end{array}\right)$,

$\mathbf{K}=\left(\begin{array}{cc}0 & 1 \\ 1 & 0\end{array}\right)$ and $\mathbf{L}=\left(\begin{array}{cc}0 & 1 \\ -1 & 0\end{array}\right)$.

$\hat{A}_{I}, \hat{A}_{J}, \hat{A}_{K}, \hat{A}_{L}, \hat{B}_{I}, \hat{B}_{J}, \hat{B}_{K}, \hat{C}_{I}, \hat{C}_{J}$ and $\hat{C}_{K}$ are 10 coefficients (scalars) 22 and

$\hat{\mathbf{D}}=-\hat{\mathbf{A}}^{\mathrm{T}}$.

The $4 \times 4$ Hamiltonian matrices define a 10-dimensional vector space. Matrices $\hat{\mathbf{B}}$ and $\hat{\mathbf{C}}$ are both symmetric, each represent a loss of a degree of freedom. Equation 16 represents a loss of four degrees of freedom. In total there is a loss of six degrees of freedom from 16 to 10 . There are thus four coefficients of $\hat{\mathbf{A}}$ (see above) and with the three each for $\hat{\mathbf{B}}$ and $\hat{\mathbf{C}}$ together they make up the 10-component coordinate vector $^{22}$

$\hat{\mathbf{v}}=\left(\hat{A}_{I} \hat{A}_{J} \hat{A}_{K} \hat{A}_{L} \hat{B}_{I} \hat{B}_{J} \hat{B}_{K} \hat{C}_{I} \hat{C}_{J} \hat{C}_{K}\right)^{\mathrm{T}}$

From a set of $N$ values of coordinate vectors $\hat{\mathbf{v}}_{1}$, $\hat{\mathbf{v}}_{2}, \ldots, \hat{\mathbf{v}}_{n}$ one can calculate a mean coordinate vector $\overline{\hat{\mathbf{v}}}$ given by ${ }^{22}$

$\overline{\hat{\mathbf{v}}}=\frac{1}{N} \sum_{n=1}^{N} \hat{\mathbf{v}}_{n}$

and a variance-covariance matrix ${ }^{22}$

$\hat{\mathbf{S}}=\frac{1}{N-1} \sum_{n=1}^{N}\left(\hat{\mathbf{v}}_{n}-\overline{\hat{\mathbf{v}}}_{n}\right)\left(\hat{\mathbf{v}}_{n}-\overline{\hat{\mathbf{v}}}_{n}\right)^{\mathrm{T}}$.

This variance-covariance matrix is a $10 \times 10$ symmetric matrix of 10 variances and 45 covariances ${ }^{14,15,22}$. The variance-covariance matrix is a compact way to represent data for the variables. Variance is a measure of the variability or spread in a set of data. Covariance indicates how two sets of ordered data vary together. Also, for a set of $N$ systems a mean transformed transference $\overline{\hat{\mathbf{T}}}$ can be obtained from ${ }^{22}$
$\overline{\hat{\mathbf{T}}}=\frac{1}{N} \sum_{n=1}^{N} \hat{\mathbf{T}}_{n}$.

Where desired, transformed transferences can be converted back to ray transferences by means of the matrix exponential $14,15,19-22$

$\tilde{\mathbf{T}}=\exp \overline{\hat{\mathbf{T}}}$.

$\tilde{\mathbf{T}}$ is the exponential-mean-logarithm-transference defined by Harris ${ }^{1,19}$ and others ${ }^{2,14,15,20-22}$. The arithmetic average or mean of the transformed transference leads to a transference which can be regarded as the transference of the average eye or cornea for the set, which is given by 20

$\tilde{\mathbf{T}}=\exp \left(\frac{1}{N} \sum_{n=1}^{N} \log \mathbf{T}_{n}\right)$

where exp represents the matrix exponential and log the principal matrix logarithm (a matrix $\mathbf{B}$ is a logarithm of a given matrix $\mathbf{A}$ if the matrix exponential of $\mathbf{B}$ is $\mathbf{A}$, that is, $e^{\mathbf{B}}=\mathbf{A}$ ). This average works for eyes because $\hat{\mathbf{T}}=\log \mathbf{T}$ is a $4 \times 4$ Hamiltonian matrix, that obeys the relation $\hat{\mathbf{T}}^{\mathrm{T}} \mathbf{E}=\mathbf{E}^{\mathrm{T}} \hat{\mathbf{T}}$.

The primary purpose of this study was to determine mean transferences for several corneas and then use these means to produce ray diagrams to study the behaviour of light rays through those corneas.

\section{Methods}

Ten healthy volunteers were recruited for this study. Subjects with a history of eye trauma, wearing contact lenses, those who had corneal or retinal disease or previous ocular surgery, for example, refractive surgery, were excluded. Data was collected from only the right eyes of subjects. The study was conducted in accordance with the Declaration of Helsinki and ethical and other approval for the study was obtained from the relevant committees of the University of Johannesburg. The full nature of the study was explained to participants and informed consent was obtained before proceeding with the measurements. All examinations were performed by the same examiner (SDM). All the measurements were obtained in photopic conditions and an attempt was made to keep the ambient luminance similar for all subjects.

With both eyes open, each subject looked at the fixation target in the Oculus Pentacam (OP) and the 
operator manually brought the image of the subject's eye into focus within the relevant circle on the instrument screen. The instrument's automatic release mode was used where the instrument automatically determined when correct focus and alignment with the corneal apex was achieved. The measurement was done in less than two seconds. During such measurements of an eye, the Scheimpflug camera in the OP rotates over 180 degrees and 25 cross-sectional or slit images of the anterior segment of the eye are obtained. Each of these 25 cross-sectional images contains 500 measurement points for the front and back corneal surfaces. After every measurement the subject removed their head from the instrument which was realigned and refocused for the next scan, thereby reducing interdependence of the readings. Subjects were also asked to blink before each measurement to distribute a smooth tear film over the cornea and 43 successive measurements of the cornea of the right eye of each subject were obtained in about 50-60 minutes per subject. A total of 43 measurements per eye was done so that possible outliers could be removed where necessary but that, at least, 40 measurements per eye would be available for further analysis. All measurements for the ten subjects were taken between 9.00 and 16.00 over a period of about eight months.

\section{Statistical analysis}

Radii of curvatures for the anterior and posterior corneal surfaces, and the central corneal thickness were obtained from the OP topography maps. The 43 simulated keratometry measurements were used to calculate the dioptric power matrices for the anterior and posterior surfaces of the cornea and transferences were calculated using equations 7 to 10 . This gave 43 transferences, $\mathbf{T}_{1}, \mathbf{T}_{2}, \ldots, \mathbf{T}_{43}$ for the cornea of each subject. From the 43 transferences, 43 transformed transferences, $\hat{\mathbf{T}}_{1}, \hat{\mathbf{T}}_{2}, \ldots, \hat{\mathbf{T}}_{43}$ were then calculated using equation 12 . The transformed transferences $(N=43)$ were expressed as coordinate vectors $\hat{\mathbf{v}}_{1}, \hat{\mathbf{v}}_{2}, \ldots, \hat{\mathbf{v}}_{43}$ and a mean coordinate vector $\overline{\hat{\mathbf{v}}}$ (equation 18) and variancecovariance $\hat{\mathbf{S}}$ (equation 19) were calculated for each eye. Using equations 20 to 22, the mean transformed or log-transference $\overline{\hat{\mathbf{T}}}$ and mean transference $\tilde{\mathbf{T}}$ were determined for each eye. (Given a set of ray transferences and as already mentioned, the simple arithmetic mean $\overline{\mathbf{T}}$ may not be a ray transference because of the symplectic condition ${ }^{13}$ but the exp-mean-logtransference $\tilde{\mathbf{T}}$ gives an average that is, in fact, a ray transference.) With the use of $\tilde{\mathbf{T}}$ as determined for each cornea, ray vector fields were generated but for brevity here only the ray vector fields for the first five subjects are included (see Figures 6 to 10).

\section{Results}

Figures 1 to 5 are stereo-pair scatter plots of the anterior and posterior corneal surface keratometric measurements obtained from the first five of the 10 subjects concerned. Each point in the scatter plot represents one keratometric measurement. The origin of each stereo-pair represents the sample mean for the cornea concerned. The $95 \%$ surfaces of constant probability density are also included. Each stereopair should be viewed by fixating at a point in front of the figure or by letting the eyes drift into an exoposture until a three-dimensional percept is obtained. For all ten subjects (and thus the five included here) there was more variation in the power of the anterior, as against posterior, corneal surface. Gillan ${ }^{24}$ found similar results for a moderately keratoconic cornea. (A model by Harris ${ }^{25,} 26$ explaining the underlying causes of corneal variation in terms of corneal curvatures rather than powers, is used in another paper ${ }^{27}$ for the same subjects. This model ${ }^{25,26}$ uses measurements of the anterior and posterior corneal surface curvatures and corneal thicknesses of the various eyes and separates out contributions relating to variation of the global curvature of the cornea from those involving local effects. For curvatures rather than powers, variation is generally greater for the posterior rather than anterior corneal surfaces ${ }^{24-27}$ and this issue will be discussed in greater detail in a later paper ${ }^{27}$ ).

a)
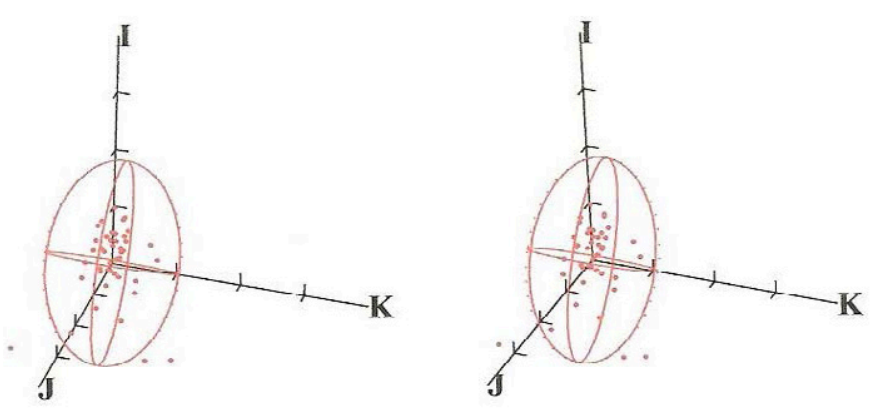
b)

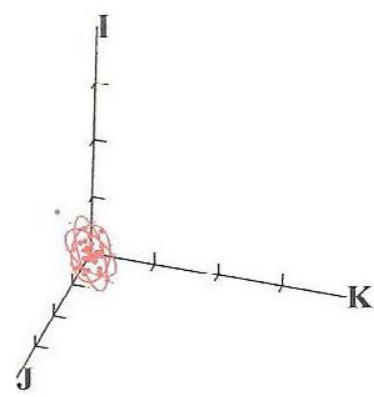

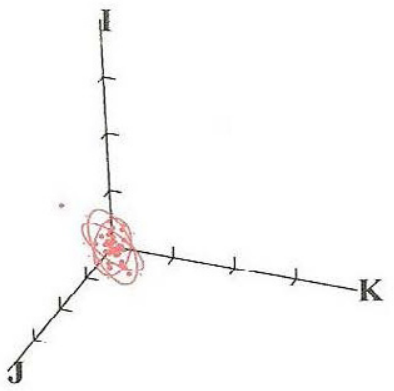

Figure 1: Stereo-pair scatter plots of symmetric dioptric power space for the anterior (a) and posterior (b) corneal keratometry for Subject 1 . The three mutually orthogonal axes labelled $\mathbf{I}, \mathbf{J}$ and $\mathbf{K}$ are the stigmatic, ortho-antistigmatic and oblique antistigmatic axes. The origin for each stereo-pair represents the sample mean concerned. The axes have ticks at intervals of $0.25 \mathrm{D}$ and the same applies to Figures 2-5. Each dot represents one measurement of the dioptric power of the anterior (a) or posterior (b) corneal surface of the eye. The stereoscopic nature of the graph is appreciated by letting the eyes drift into an exo-posture to form a single 3-dimensional percept.

a)

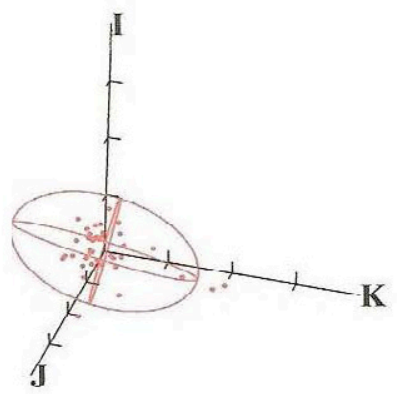

b)
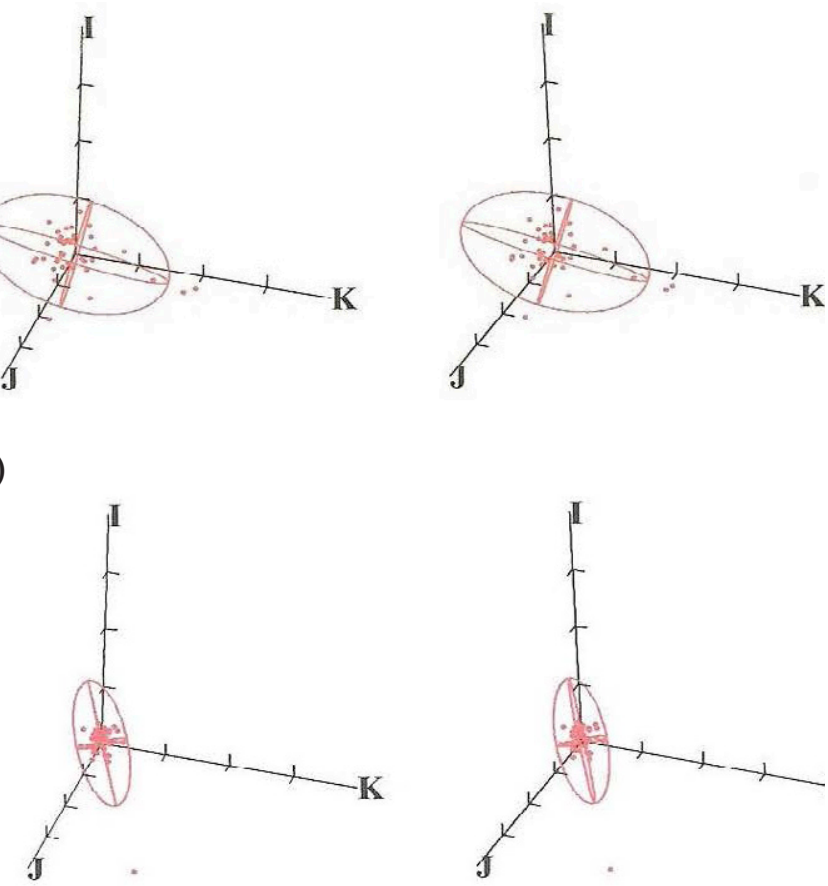

Figure 2: Stereo-pair scatter plot of symmetric dioptric power space for the anterior (a) and posterior (b) corneal keratometry for Subject 2. The origin for each stereo-pair is the respective sample mean.

a)

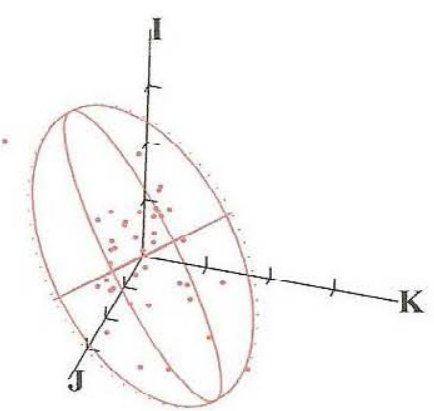

b)
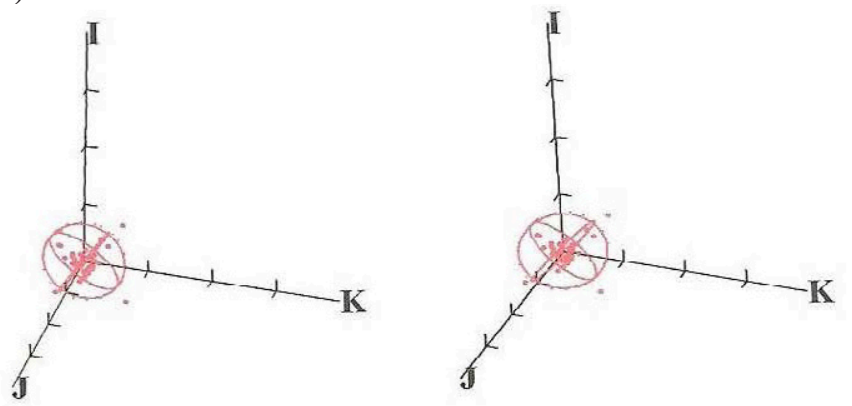

Figure 3: Stereo-pair scatter plots in symmetric dioptric power space for the anterior (a) and posterior (b) corneal keratometry for Subject 3.

a)
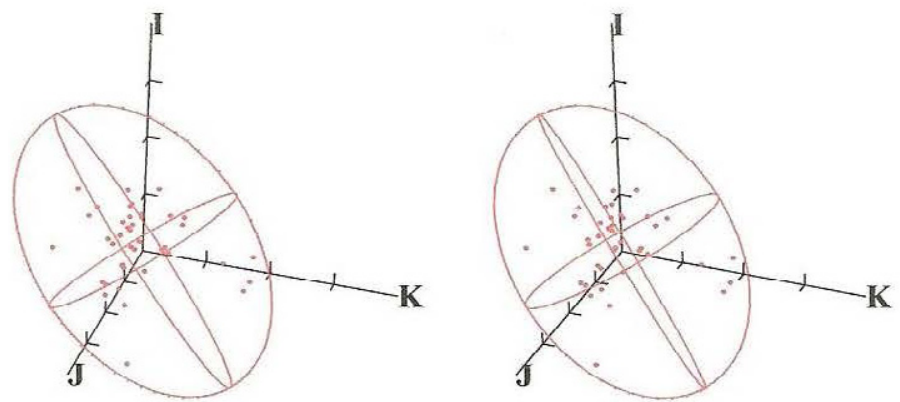

b)
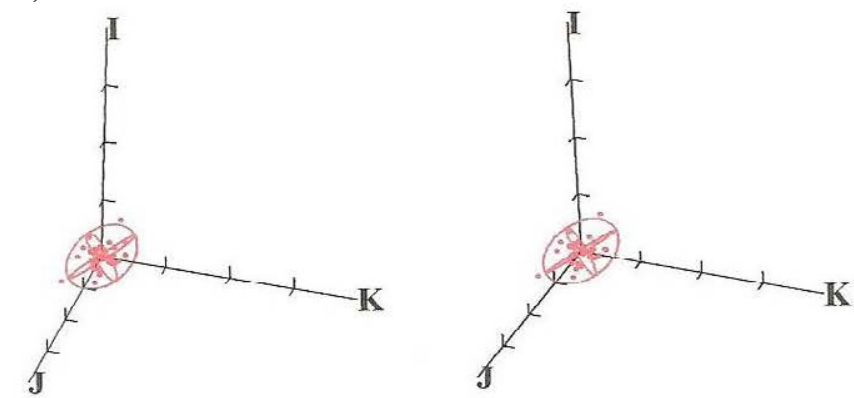

Figure 4: Stereo-pair scatter plots in symmetric dioptric power space for the anterior (a) and posterior (b) corneal keratometry for Subject 4 .

a)
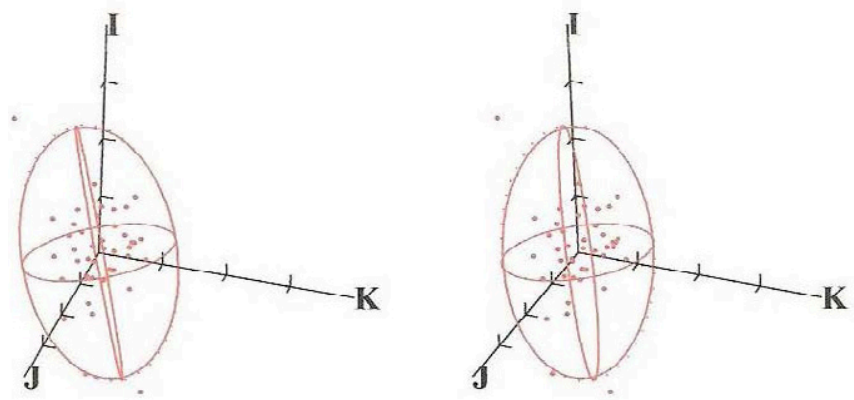
b)
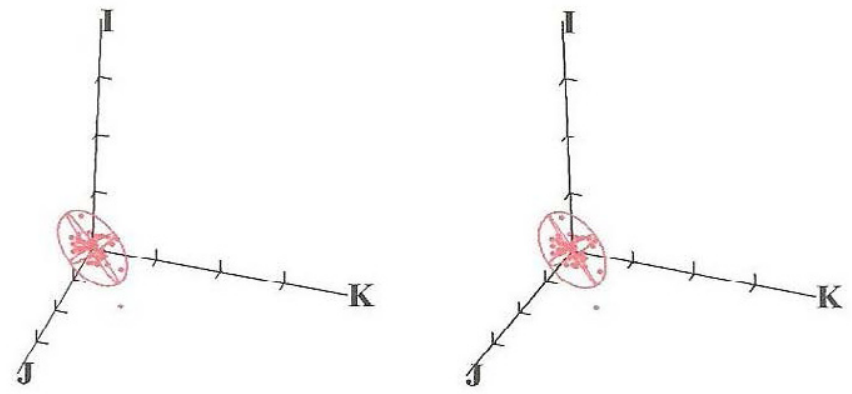

Figure 5: Stereo-pair scatter plots in symmetric dioptric power space for the anterior (a) and posterior (b) corneal keratometry for Subject 5.

Table 1 shows the averages of transformed $\overline{\hat{\mathbf{T}}}$ and recovered, or untransformed, $\tilde{\mathbf{T}}$ transferences of the 10 subjects. The logm function (in Matlab) transformed the ray transferences into Hamiltonian matrices. Once in Hamiltonian space, arithmetic means were computed. These means were in turn transformed back to ray transferences via the expm function. These are the average transferences resulting from the application of the exp-mean-log. The mean coordinate vectors for the 10 eyes are shown in Table 2 while Table 3 shows the 10x10 variancecovariances $\hat{\mathbf{S}}$ of Subject 1 only (the same was done for all subjects but to save space only the matrix $\hat{\mathbf{S}}$ for Subject 1 is included here). Since the matrix $\hat{\mathbf{S}}$ is symmetric, there are 10 variances (shown in bold along the diagonal) and 45 distinct covariances (for example, all entries below the diagonal).

Table 1. Averages of transformed ( $\overline{\hat{\mathbf{T}}}$ ) in Hamiltonian space and recovered ( $\tilde{\mathbf{T}})$ mean transferences for the corneas of the 10 subjects are indicated. Subscripts from 1 to 10 are used to indicate the ten different subjects. Units have been omitted to save space but for each transference disjugacy $\mathbf{B}$ (the top-right four numbers) has the unit metres while the sub-matrix $\mathbf{C}$ (bottom-left four entries) is in dioptres. All other entries are unitless. The mean transformed transferences are determined in Hamiltonian space and the mean transferences are recovered using the matrix exponential.

\section{Average Transformed Transferences}

$$
\begin{aligned}
& \overline{\hat{\mathbf{T}}}_{1}=\left(\begin{array}{cccc}
-0.0123 & -0.0000 & 0.0005 & 0.0000 \\
-0.0000 & -0.0125 & 0.0000 & 0.0005 \\
-41.9877 & 0.0000 & 0.0123 & 0.0000 \\
0.0000 & -42.1148 & 0.0000 & 0.0125
\end{array}\right) \\
& \overline{\hat{\mathbf{T}}}_{2}=\left(\begin{array}{cccc}
-0.0107 & 0.0001 & 0.0004 & -0.0000 \\
0.0001 & -0.0110 & -0.0000 & 0.0004 \\
-42.4311 & -0.0001 & 0.0107 & -0.0001 \\
-0.0001 & -42.9482 & -0.0001 & 0.0110
\end{array}\right) \\
& \overline{\hat{\mathbf{T}}}_{3}=\left(\begin{array}{cccc}
-0.0120 & 0.0000 & 0.0004 & -0.0000 \\
0.0000 & -0.0120 & -0.0000 & 0.0004 \\
-41.5744 & -0.0000 & 0.0120 & -0.0000 \\
-0.0000 & -41.5460 & -0.0000 & -0.0120
\end{array}\right) \\
& \overline{\hat{\mathbf{T}}}_{4}=\left(\begin{array}{cccc}
-0.0109 & 0.0000 & 0.0004 & -0.0000 \\
0.0000 & -0.0112 & -0.0000 & 0.0004 \\
-40.7042 & -0.0000 & 0.0109 & -0.0000 \\
-0.0000 & -41.2433 & -0.0000 & 0.0112
\end{array}\right) \\
& \overline{\hat{\mathbf{T}}}_{6}=\left(\begin{array}{cccc}
\mathbf{\hat { \mathbf { T } }} \\
-0.0117 & 0.0000 & 0.0004 & -0.0000 \\
0.0000 & -0.0118 & -0.0000 & 0.0004 \\
-42.0682 & -0.0000 & -0.0117 & -0.0000 \\
-0.0000 & -42.0409 & -0.0000 & 0.0118 \\
-0.0106 & 0.0001 & 0.0004 & -0.0000 \\
0.0001 & -0.0104 & -0.0000 & 0.0004 \\
-42.9205 & -0.0001 & 0.0106 & -0.0001 \\
-0.0001 & -41.8859 & -0.0001 & 0.0104
\end{array}\right)
\end{aligned}
$$

\section{Recovered Mean Transferences}

$$
\tilde{\mathbf{T}}_{1}=\left(\begin{array}{cccc}
0.9782 & -0.0000 & 0.0005 & 0.0000 \\
-0.0000 & 0.9779 & 0.0000 & 0.0005 \\
-41.8538 & 0.0000 & 1.0027 & 0.0000 \\
0.0000 & -41.9801 & 0.0000 & 1.0029
\end{array}\right)
$$$$
\tilde{\mathbf{T}}_{2}=\left(\begin{array}{cccc}
0.9810 & 0.0000 & 0.0004 & -0.0000 \\
0.0000 & 0.9807 & -0.0000 & 0.0004 \\
-42.3138 & -0.0000 & 1.0024 & -0.0001 \\
-0.0000 & -42.8280 & -0.0001 & 1.0025
\end{array}\right)
$$$$
\tilde{\mathbf{T}}_{3}=\left(\begin{array}{cccc}
0.9789 & 0.0000 & 0.0004 & -0.0000 \\
0.0000 & 0.9788 & -0.0000 & 0.0004 \\
-41.4468 & -0.0000 & 1.0027 & -0.0000 \\
-0.0000 & -41.4186 & -0.0000 & 1.0028
\end{array}\right)
$$$$
\tilde{\mathbf{T}}_{4}=\left(\begin{array}{cccc}
0.9807 & 0.0000 & 0.0004 & -0.0000 \\
0.0000 & 0.9803 & -0.0000 & 0.0004 \\
-40.5900 & -0.0000 & 1.0025 & -0.0000 \\
-0.0000 & -41.1261 & -0.0000 & 1.0026
\end{array}\right)
$$$$
\tilde{\mathbf{T}}_{5}=\left(\begin{array}{cccc}
0.9793 & 0.0000 & 0.0004 & -0.0000 \\
0.0000 & 0.9792 & -0.0000 & 0.0004 \\
-41.9419 & -0.0000 & 1.0027 & -0.0000 \\
-0.0000 & -41.9147 & -0.0000 & 1.0028
\end{array}\right)
$$$$
\tilde{\mathbf{T}}_{6}=\left(\begin{array}{cccc}
0.9813 & 0.0001 & 0.0004 & -0.0000 \\
0.0001 & 0.9817 & -0.0000 & 0.0004 \\
-42.8038 & -0.0000 & 1.0024 & -0.0001 \\
-0.0000 & -41.7747 & -0.0001 & 1.0024
\end{array}\right)
$$ 


\begin{tabular}{|c|c|c|c|c|c|c|c|c|c|}
\hline \multirow{4}{*}{$\overline{\hat{\mathrm{T}}}_{7}=$} & -0.0097 & 0.0000 & 0.0004 & -0.0000 & \multirow{4}{*}{$\tilde{\mathbf{T}}_{7}=$} & 0.9827 & 0.0000 & 0.0004 & -0.0000 \\
\hline & 0.0000 & -0.0098 & -0.0000 & 0.0004 & & 0.0000 & 0.9827 & -0.0000 & 0.0004 \\
\hline & -42.7257 & -0.0000 & 0.0097 & -0.0000 & & -42.6183 & -0.0000 & 1.0022 & -0.0000 \\
\hline & -0.0000 & -42.5672 & -0.0000 & 0.0098 & & -0.0000 & -42.4606 & -0.0000 & 1.0023 \\
\hline \multirow{4}{*}{$\overline{\hat{\mathbf{T}}}_{8}=$} & -0.0104 & 0.0000 & 0.0004 & -0.0000 & \multirow{4}{*}{$\tilde{\mathbf{T}}_{8}=$} & 0.9816 & 0.0000 & 0.0004 & -0.0000 \\
\hline & 0.0000 & -0.0107 & -0.0000 & 0.0004 & & 0.0000 & 0.9811 & -0.0000 & 0.0004 \\
\hline & -42.1083 & -0.0000 & 0.0104 & -0.0000 & & -41.9956 & -0.0000 & 1.0023 & -0.0000 \\
\hline & -0.0000 & -43.0703 & -0.0000 & 0.0107 & & -0.0000 & -42.9525 & -0.0000 & 1.0025 \\
\hline \multirow{4}{*}{$\overline{\hat{\mathbf{T}}}_{9}=$} & -0.0098 & 0.0000 & 0.0004 & -0.0000 & \multirow{4}{*}{$\tilde{\mathbf{T}}_{9}=$} & 0.9827 & 0.0000 & 0.0004 & -0.0000 \\
\hline & 0.0000 & -0.0101 & -0.0000 & 0.0004 & & 0.0000 & 0.9822 & -0.0000 & 0.0004 \\
\hline & -40.7345 & -0.0000 & 0.0098 & -0.0000 & & -40.6317 & -0.0001 & 1.0022 & -0.0000 \\
\hline & -0.0000 & -41.7823 & -0.0000 & 0.00101 & & -0.0001 & -41.6741 & -0.0000 & 1.0023 \\
\hline \multirow{4}{*}{$\hat{\mathbf{T}}_{10}=$} & -0.0104 & -0.0000 & 0.0006 & 0.0000 & \multirow{4}{*}{$\tilde{\mathbf{T}}_{10}=$} & 0.9815 & -0.0000 & 0.0004 & 0.0000 \\
\hline & -0.0000 & -0.0106 & 0.0000 & 0.0004 & & -0.0000 & 0.9814 & 0.0000 & 0.0004 \\
\hline & -42.2007 & 0.0000 & 0.0104 & 0.0000 & & -42.0870 & 0.0000 & 1.0023 & 0.0000 \\
\hline & 0.0000 & -42.3581 & 0.0000 & 0.0106 & & 0.0000 & -42.2436 & 0.0000 & 1.0024 \\
\hline
\end{tabular}

Table 2. Mean coordinate vectors ( $\overline{\hat{\mathbf{v}}}$ ) from transformed transferences of ten subjects. Subscripts from 1 to 10 are used to indicate the ten different subjects. In each vector, the first four entries are unit-less, the second three entries are in metres while the last three entries are in dioptres.

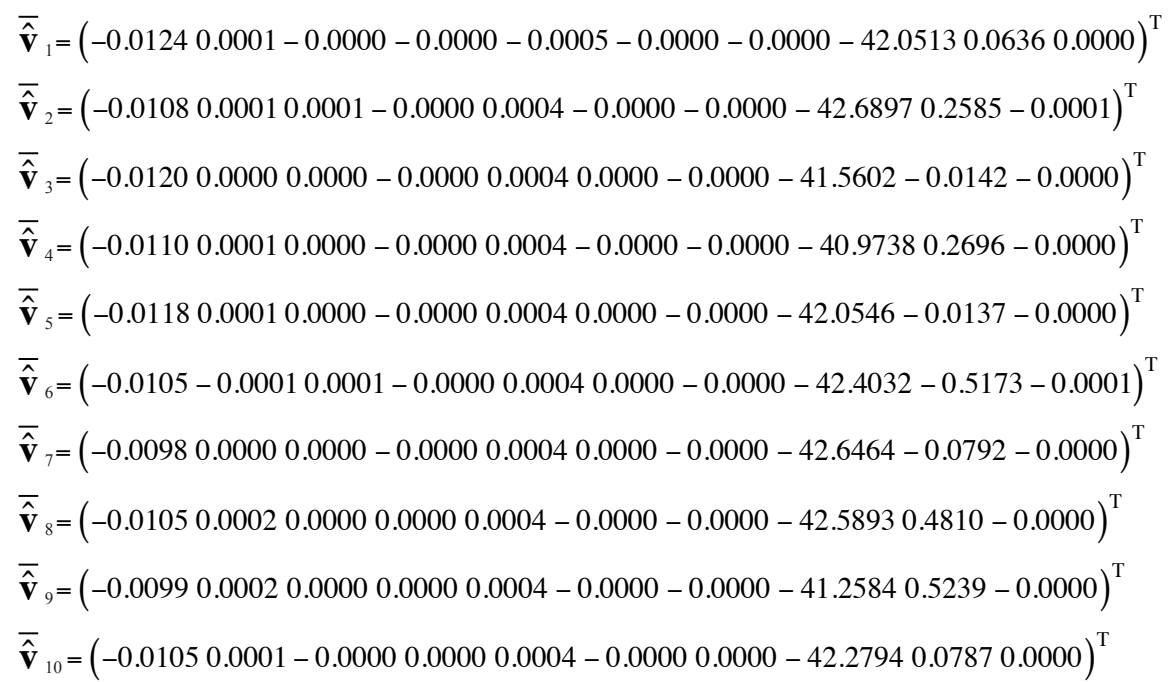

Table 3. The sample variance-covariance matrix $\hat{\mathbf{S}}$ for the coordinate vectors $(N=43)$ for the cornea of Subject 1 is indicated. Units have been omitted to save space. They are metres for $\hat{\mathbf{B}}$ (see blue block), dioptres for $\hat{\mathbf{C}}$ (green block) and $\hat{\mathbf{A}}$ is unit-less (red block). The matrix is symmetric so we are interested only in the 10 variances (in bold, top left to bottom right) and the 45 covariances (for example, below the diagonal). Most of the covariances are almost zero. The entries in the red block are the variances and covariances of $\hat{\mathbf{S}}_{\hat{\mathbf{A}}}$ while those in the blue and green blocks are for $\hat{\mathbf{S}}_{\hat{\mathbf{B}}}$ and $\hat{\mathbf{S}}_{\hat{\mathbf{C}}}$ respectively. Each of the entries of $\hat{\mathbf{S}}$ has been pre-multiplied by 103 and hence the 10-3 that follows the matrix below.

$\hat{\mathbf{S}}=\left(\begin{array}{cccc|cccccc}\mathbf{0 . 0 0 0 0} & -0.0000 & 0.0000 & -0.0000 & -0.0000 & -0.0000 & -0.0000 & 0.0011 & 0.0003 & 0.0000 \\ -0.0000 & \mathbf{0 . 0 0 0 0} & 0.0000 & -0.0000 & -0.0000 & -0.0000 & -0.0000 & -0.0034 & -0.0010 & -0.0000 \\ 0.0000 & 0.0000 & \mathbf{0 . 0 0 0 0} & -0.0000 & -0.0000 & -0.0000 & -0.0000 & -0.0003 & 0.0004 & 0.0000 \\ -0.0000 & -0.0000 & -0.0000 & \mathbf{0 . 0 0 0 0} & 0.0000 & 0.0000 & 0.0000 & -0.0000 & -0.0000 & 0.0000 \\ \hline-0.0000 & -0.0000 & -0.0000 & 0.0000 & \mathbf{0 . 0 0 0 0} & 0.0000 & 0.0000 & 0.0001 & -0.0001 & 0.0000 \\ -0.0000 & -0.0000 & -0.0000 & 0.0000 & 0.0000 & \mathbf{0 . 0 0 0 0} & 0.0000 & 0.0000 & -0.0000 & 0.0000 \\ -0.0000 & -0.0000 & -0.0000 & 0.0000 & 0.0000 & 0.0000 & \mathbf{0 . 0 0 0 0} & 0.0000 & -0.0000 & 0.0000 \\ 0.0011 & -0.0034 & -0.0003 & -0.0000 & 0.0001 & 0.0000 & 0.0000 & \mathbf{2 9 . 7 1 6 5} & -4.2038 & 0.0003 \\ 0.0003 & -0.0010 & 0.0004 & -0.0000 & -0.0001 & -0.0000 & -0.0000 & -4.2038 & \mathbf{8 . 0 2 0 6} & -0.0004 \\ 0.0000 & -0.0000 & 0.0000 & 0.0000 & 0.0000 & 0.0000 & 0.0000 & 0.0003 & -0.0004 & \mathbf{0 . 0 0 0 0}\end{array}\right) \times 10^{-3}$


Figures 6 to 10 show the ray vector fields or diagrams for the first five of 10 subjects. Each of the figures represents the cornea as an optical system. In Figure 6a light is considered to be travelling into the page and the optical axis is perpendicular to the page and is marked with a small cross $(+)$. In the entrance and exit planes, a pattern of 25 coloured dots represents the incident positions of 25 rays from a distant object arranged in a regular grid. Twenty-five small coloured circles with bars (in the exit plane) represent emergent positions and reduced inclinations of the 25 rays. (Note the bars indicate ray position and direction but not magnitude.) The entrance and exit planes are also superimposed in Figure 6a to demonstrate for each ray the manner by which its incident state is transformed to its emergent state. In Figure 6b, the 25 rays of Figure $6 \mathrm{a}$ are shown in a stereo-pair to more easily visualize in three dimensions the ray behaviour and the changes from incident to emergent states.

In Figure 6 a the matrix $\tilde{\mathbf{T}}$ is the linear map that assigns to each incident ray vector $\gamma_{o}$ an emergent ray

a)

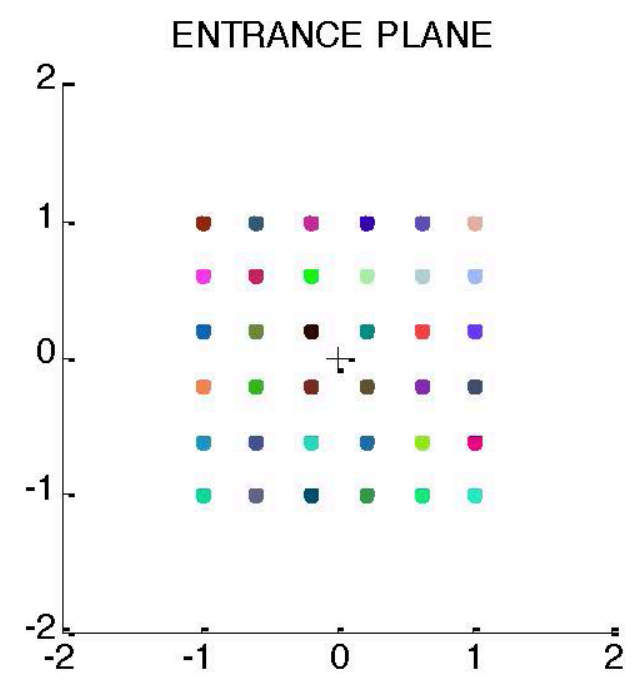

ENTRANCE AND EXIT PLANES SUPERIMPOSED vector $\boldsymbol{\gamma}$. This mean transference, $\tilde{\mathbf{T}}$ operates on, or modifies, the incident rays to give the emergent rays. The matrix $\mathbf{F}_{0}$ is the corneal plane refractive power and the magnitude of these corneal plane powers are very large ( $>2000$ D for Subject 1 in Figure 6) relating to the central thickness of the cornea being only about $0.5 \mathrm{~mm} . \mathbf{F}_{0}$ is essentially the power of a thin lens immediately in front of the cornea which compensates for the system ametropia; assuming we regard the optical system as that of the cornea in isolation, that is, the emergent plane is located just before the posterior surface of the cornea. (Matrix $\mathbf{F}_{0}$ does not depend directly on the power of the cornea.) The light rays bend or converge very dramatically to reach the posterior corneal surface or exit plane given that the cornea (considered as optical system in isolation is only about $0.5 \mathrm{~mm}$ thick). Also indicated in the figure is the matrix $\mathbf{F}$ (the dioptric power of the cornea) and at about $42 \mathrm{D}$ is not atypical of what might be found for corneas of young subjects.

In Figures 7-10 only stereo-pairs are used to indicate ray behaviour through the corneas of subjects ${ }^{2-5}$.

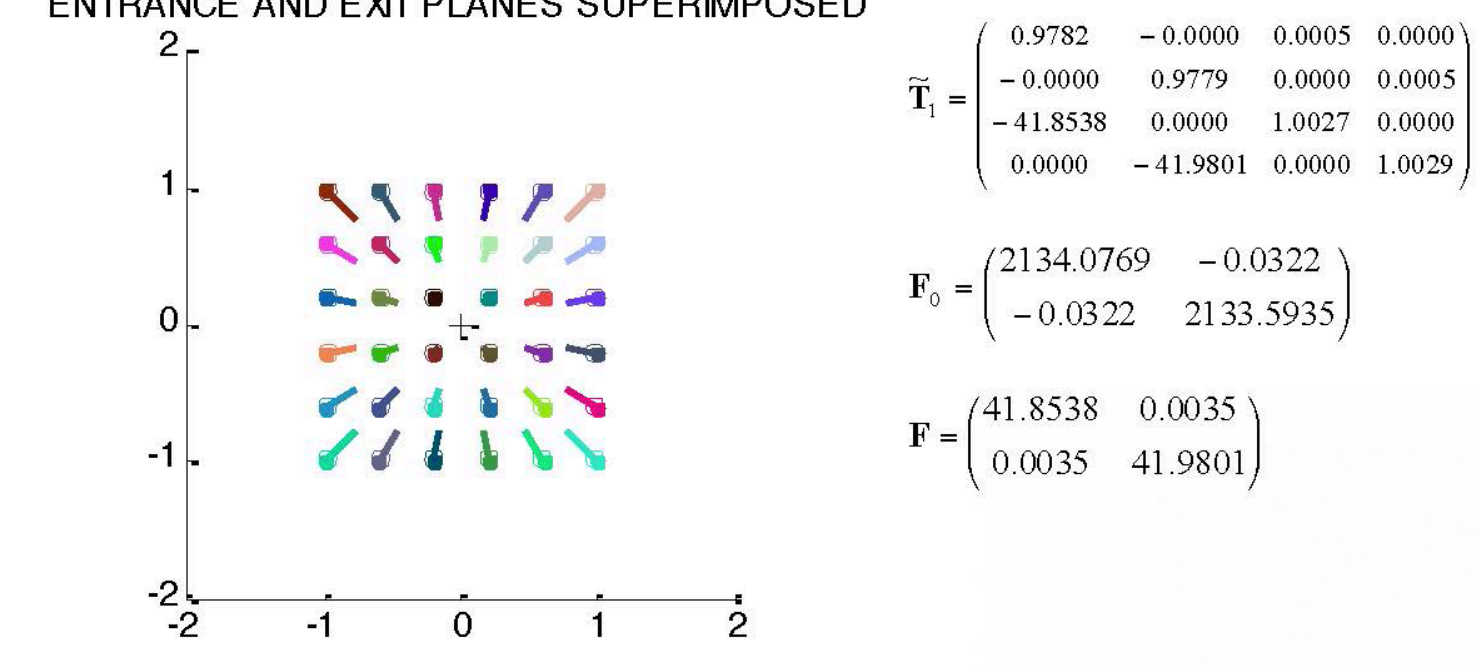

EXIT PLANE

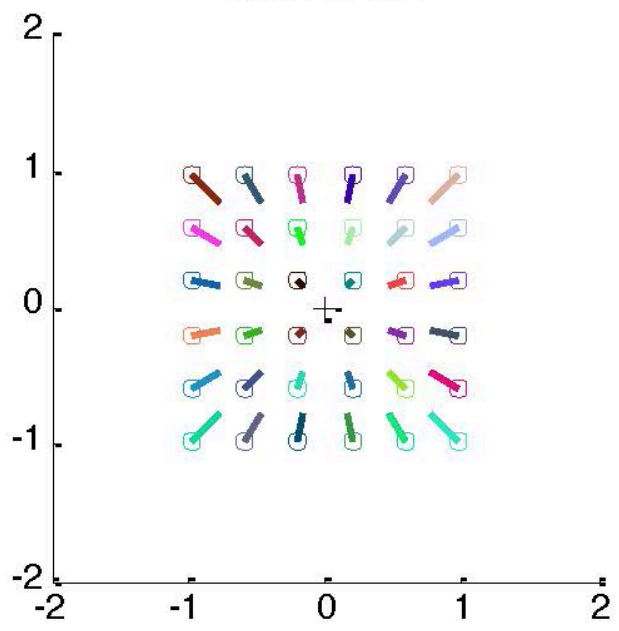




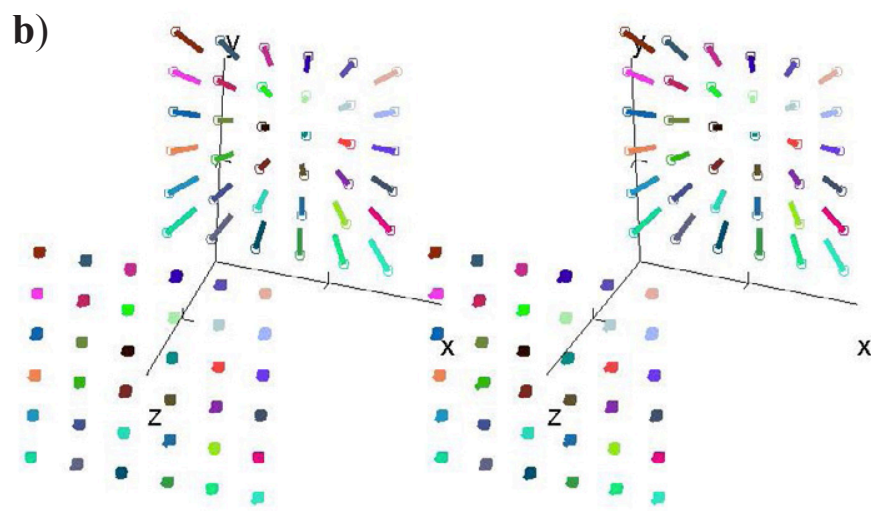

Figure 6. Interpretation of light rays traversing the cornea of Subject 1. (a) The 25 coloured dots represent the incident rays in the entrance plane and the corresponding emergent rays are represented with the same colours but by circles with bars. The optical axis is marked with a cross. The optical axis itself represents any ray through the system that is not deviated. So, any ray can serve as an optical axis. The last row and last column of $\tilde{\mathbf{T}}$ indicate that the system is centred. $\mathbf{F}_{0}$ is the corneal plane refraction and is very large indicating that rays converge markedly when passing through the entrance plane or anterior corneal surface.. The power of the cornea is represented by F. All light rays converge toward the optical axis as shown with coloured open circles and bars in the exit plane. (b) Stereo-pair scatter plot of the ray vectors in three-dimensions. The z-axis represents the optical axis and the separation between the entrance and exit planes is exaggerated for ease of inspection.

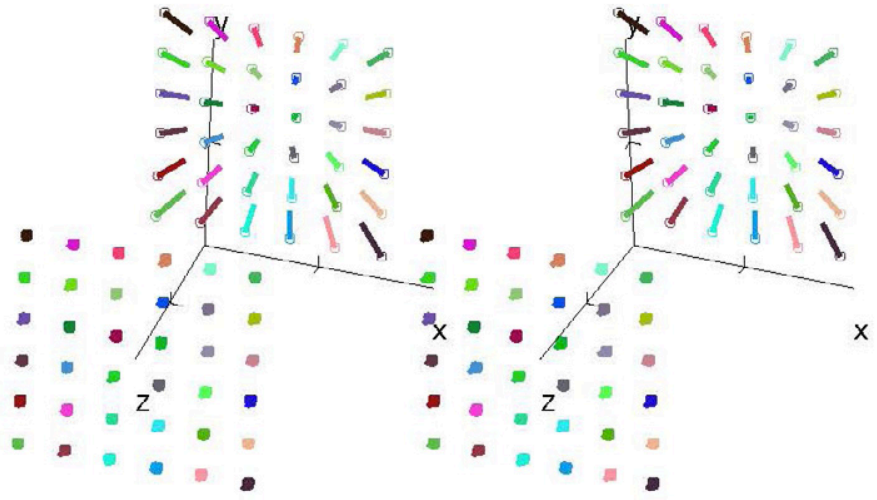

Figure 7. Stereo-pair scatter plot of the ray vectors traversing the cornea of Subject 2 in three-dimensions. Again the z-axis represents the optical axis and the separation between the entrance and exit planes is exaggerated for ease of inspection.

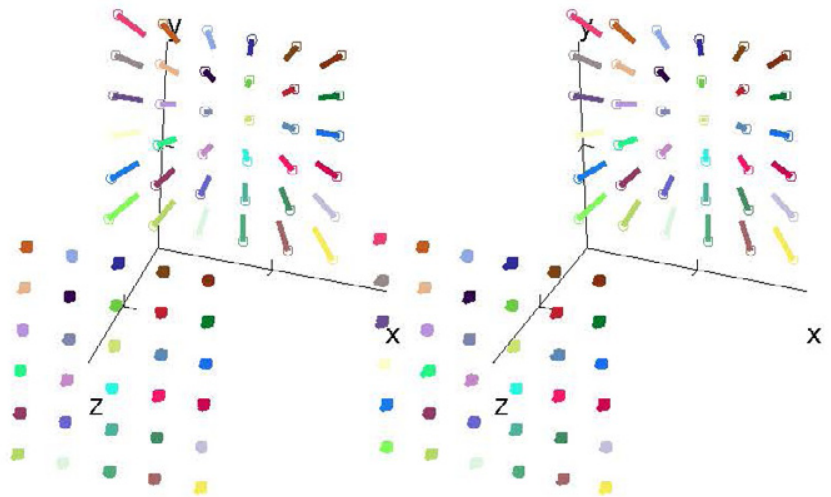

Figure 8. Stereo-pair scatter plot of the ray vectors traversing the cornea of Subject 3 in three-dimensions.

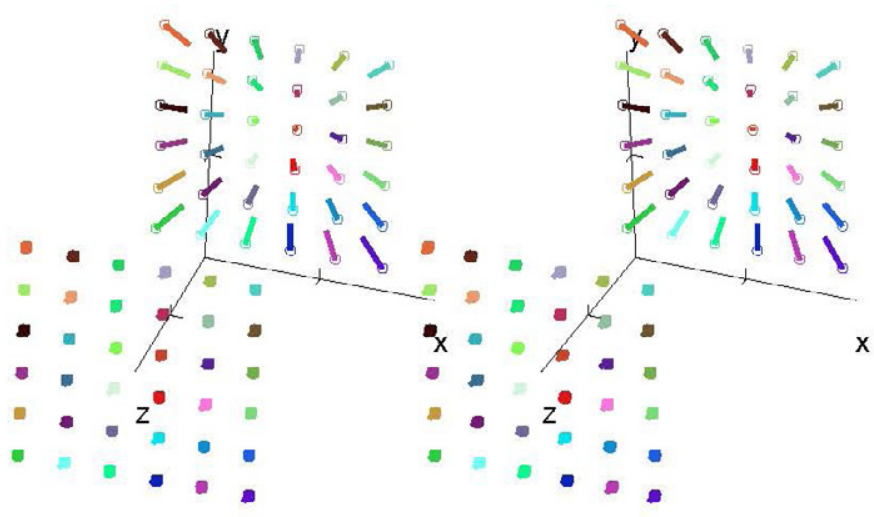

Figure 9. Stereo-pair scatter plot of the ray vectors traversing the cornea of Subject 4.

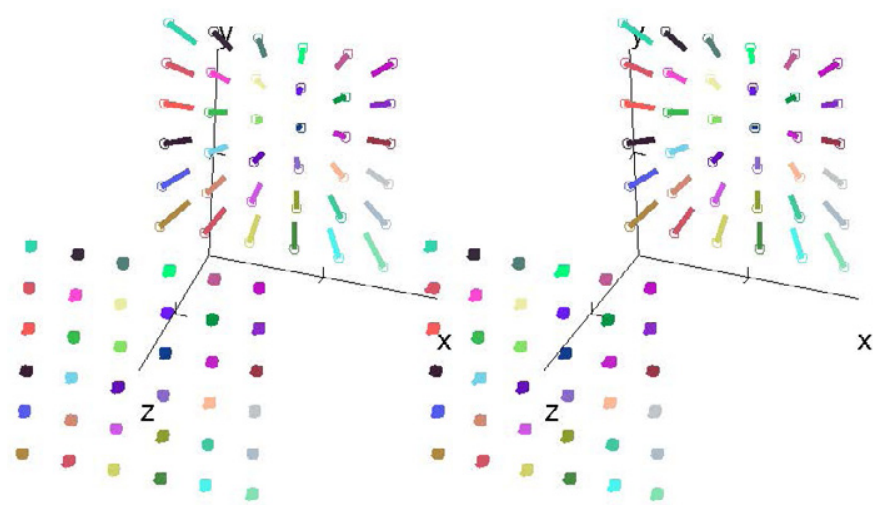

Figure 10. Stereo-pair scatter plot of the ray vectors traversing the cornea of Subject 5 .

\section{Discussion}

The general and unique definition of power, and understanding the behaviour of light rays in relation to an optical system, are both found in terms of the ray transference $\mathbf{T}$ which is a symplectic matrix ${ }^{1,9}$. Symplecticity implies particular relationships among the fundamental linear optical properties of an optical system ${ }^{18}$. The product of symplectic matrices is symplectic, that is, symplectic matrices are closed under multiplication but they are not closed under scalar multiplication. Also, symplectic matrices are not closed in general under addition and thus addition of two symplectic matrices will usually return a matrix that is itself not symplectic. For this reason, an arithmetic average of symplectic matrices is not symplectic and this creates problems determining, for example, a mean cornea for one or more eyes such as are provided in this paper. But the principal matrix logarithm of a symplectic matrix is a Hamiltonian matrix and the matrix exponential of a Hamiltonian matrix is 
symplectic ${ }^{20}$ and such matrices can thus be used to determine means. The method is implemented in Matlab where the logarithm function transforms ray transferences into Hamiltonian matrices and allows the mean, variances and covariances of such transferences to be calculated. Thereafter the exponential function transforms the mean $\log$ transference in Hamiltonian space back into the sample mean ray transference. In other words, for a sample of ray transferences (here $\mathbf{T}_{1}$ to $\mathbf{T}_{43}$ ) the mean ray transference $(\tilde{\mathbf{T}})$ recovered from the mean Hamiltonian matrix $(\overline{\hat{\mathbf{T}}})$ is the average cornea of the eye concerned. In Table 1 the mean transferences are indicated for the corneas of the ten right eyes that were studied for this paper.

For a Hamiltonian matrix (see equation 13) the offdiagonal sub-matrices $\hat{\mathbf{B}}$ and $\hat{\mathbf{C}}$ are symmetric and one diagonal sub-matrix ( $\hat{\mathbf{A}}$ ) is the negative of the transpose of the other $\left(-\hat{\mathbf{D}}^{\mathrm{T}}\right)^{22}$. A $4 \times 4$ Hamiltonian matrix represents a loss of a degree of freedom $(d f)$ each from the off-diagonal sub-matrices and a loss of four degrees of freedom from one of the diagonal sub-matrix. There is a loss of six degrees of freedom from 16 to 10 . The $4 \times 4$ symplectic matrix for a centered optical system, such as those described in this paper, has only 10 degrees of freedom instead of 16 . So the $4 \times 4$ Hamiltonian matrices define a 10-dimensional vector space. But effective graphical representation of a 10-dimensional vector space is impossible without resorting to various simplifications that do not allow for an entirely satisfactory representation of the 10-dimensional space ${ }^{15}$. However, one can work in the 10-dimensional space mathematically and calculate arithmetic means $(\overline{\hat{\mathbf{T}}})$ and $10 \times 10$ variance-covariance matrices $(\hat{\mathbf{S}})$ in Hamiltonian space and thereby determine a mean transference $(\tilde{\mathbf{T}})$ that suitably describes the sample or transferences or optical systems concerned ${ }^{15,22}$.

The mean ray transference $(\tilde{\mathbf{T}})$ can be regarded as an operator that transforms or operates on the incident positions and directions of rays $\left(\mathbf{y}_{0}\right.$ and $\left.\boldsymbol{\alpha}_{0}\right)$ to give the emergent and positions and directions of rays ( $\mathbf{y}$ and $\boldsymbol{\alpha}$ ). Thus when a ray traverses an optical system the state of the ray is changed from its incident state to its emergent state. This quantitative change is represented by the ray transference of the system and dioptric power, for example, is explained in terms of what the optical system does to rays passing through the system ${ }^{9-12}$. Figures 6 to 10 use the mean transferences $(\tilde{\mathbf{T}})$ of the different corneas, or optical systems, of ten eyes to show the effects of such systems on the emergent positions and directions of defined rays from a distant point image incident on the systems concerned. These figures represent what are known as vector ray fields and explain how the rays are affected by the optical systems concerned.

The dioptric power of the system can be defined as the negative of divergence $(\mathbf{F}=-\mathbf{C})$ of the mean transference for the system (see Figure 6a where $\mathbf{F}$ is indicated) for Subject 1 . The dioptric power of the system is the negative of the effect of the incident position of a ray on its emergent reduced inclination. Because the system has power $\mathbf{F}$ there is a contribution $-\mathbf{F} \mathbf{y}_{0}$ associated with incident position $\mathbf{y}_{0}$ to an emergent reduced inclination $\boldsymbol{\alpha}$. When the entrance and exit planes are superimposed, such as in the bottomleft section of Figure $6 \mathrm{a}$, then coloured points or dots represent incident positions while circles and short lines represent emergent ray positions and directions. In the stereo-pair of Figures 6b, and also for those of Figures 7 to 10 for the different eyes in this sample, both incident and emergent positions and their incident and emergent directions are represented graphically in the incident and exit planes which are located at the anterior corneal surfaces and just in front of the posterior corneal surfaces respectively of the eyes involved. So, for each subject here the optical system consists only of the cornea in isolation of the rest of the eye concerned.

Table 3 shows the variation (of coordinate vectors from the transformed transferences of Subject 1) via a $10 \times 10$ variance-covariance matrix $\hat{\mathbf{S}}$ in $\log$ transference or Hamiltonian space. (The mean coordinate vector for this eye is provided in Table 2 but for each of the 43 transformed transferences of this eye there would be a corresponding coordinate vector, and since these vectors are somewhat different to one another that variation is described in the variance-covariance matrix $\hat{\mathbf{S}}$ for the eye.) The Hamiltonian space has three sub-spaces (but in general it has five subspaces ${ }^{21,22}$ ). The fact that most entries in the variance-covariance of Table 3 are close to zero is a consequence of the cornea being a system that is "almost" thin. If the cornea was a thin system then all entries would be zero except the lower-right $3 \times 3$ block representing the variation of $\hat{\mathbf{C}}$. 


\section{Conclusion}

In summary, the interpretation presented in this study describes transferences in terms of what the cornea does to rays traversing it within the limitations of paraxial optics. The cornea operates on rays according to equation 1 . From the 43 measurements of corneal powers and central thicknesses for each subject, there are thus 43 equations in the unknown matrix T. The optical system, namely the subject's cornea, varies and there are 43 transferences for which we require the determination of a mean ray transference that adequately represents the average or mean optical system (or cornea) for the subject concerned. To this end average transferences $\tilde{\mathbf{T}}$ as well as ray vector fields (Figures 6-10) were determined and represented graphically for Subect 1 and others thus permitting us to better understand the behaviour of paraxial light rays through the optical systems as defined.

Light rays near the optical axis enter the cornea at the entrance plane and leave the system at the exit plane. When the rays traverse the system (or cornea here) the state of the ray is changed from its incident to its emergent state by the system ray transference. We are thus only interested in rays near the optical axis of the system. The optical axis itself represents any ray that goes through the cornea undeviated ${ }^{28}$. Dealing with rays further from the optical axis becomes much more difficult and complicated. Figures 6 to 10 of ray vector fields for several eyes aid our interpretation of a defined set of rays reaching the incident plane from a distant object point and such diagrams clearly show what happens when such light rays enter the involved cornea. Although this paper concentrates on the cornea alone as the optical system it would be possible to trace rays through the whole eye but this would require the measurement of other variables such as the axial thickness and surface powers of the crystalline lens, and also the axial length of the vitreous chamber. The methods used here however allow for a better understanding of paraxial ray tracing and the nature of the interaction of the system (of the whole eye) with light and so future research should perhaps be applied to measuring such parameters directly for a few eyes and then applying the analytical and graphical methods as used in this paper to said measurements.

Many of the ideas and methods as described and used in this paper may seem difficult at first glance but they are essential if we want to properly understand the interaction of the eye and light in its environment. The optics of the cornea and eye (both of which are effectively thick, as against thin, optical systems) are in many ways more complicated than one might anticipate and this is particularly so due to the existence of astigmatism. Nevertheless, methods that involve ray transferences are available to begin to better understand these intriguing aspects of vision, light and the eye.

\section{Acknowledgement}

Thanks are extended to Professor WF Harris for comment and discussions relating to several ideas and issues as described in this paper.

\section{References}

1. Harris WF. The average eye. Ophthal Physiol Opt 200424 580-585.

2. Van Gool RD and Harris WF. The concept of the average eye. S Afr Optom 200564 38-43.

3. Harris WF and van Gool RD. First-order characteristic matrices of optical systems. S Afr Optom 200463 142-146.

4. Harris WF. Astigmatic optical systems with separated and prismatic or noncoaxial elements: system matrices and system vectors. Optom Vis Sci 199370 545-551.

5. Keating MP. A system matrix for astigmatic optical systems: I. Introduction and dioptric power relations. Am J Optom Physiol Opt 198158 810-819.

6. Keating MP. A system matrix for astigmatic optical systems: II. Corrected systems including an astigmatic eye. Am J Optom Physiol Opt 198158 919-929.

7. Keating MP. Advantage of a block matrix formulation for an astigmatic system. Am J Optom Physiol Opt 198259851 857.

8. Harris WF. Paraxial ray tracing through noncoaxial astigmatic optical systems, and a 5x5 augmented system matrix. Optom Vis Sci 199471 282-285.

9. Harris WF. Dioptric power: its nature and its representation in three- and four-dimensional space. Optom Vis Sci 1997 74 480-499.

10. Harris WF. A unified paraxial approach to astigmatic optics. Optom Vis Sci 199976 480-499.

11. Harris WF. Magnification, blur, and ray state at the retina for the general eye with and without a general optical instrument in front of it. I. Distance object. Optom Vis Sci 2110 78 888-900.

12. Harris WF. The four fundamental properties of Gaussian optical systems including the eye. S Afr Optom 199958 69-79. 
13. Harris WF. Symplecticity in visual optics. S Afr Optom 2002 61 97-100.

14. Mathebula SD. Quantitative analysis of the linear optical character of the anterior segment of the eye. Doctoral thesis in the Department of Optometry, University of Johannesburg, South Africa, 2010.

15. Mathebula SD, Rubin A and Harris WF. Quantitative analysis in Hamiltonian space of the transformed ray transferences of a cornea. S Afr Optom 200766 68-76.

16. Harris WF. Ray vector fields, prismatic effect, and thick astigmatic optical systems. Optom Vis Sci 199673 418-423.

17. Keating MP. A matrix formulation of spectacle magnification. Ophthal Physiol Opt 19822 145-158.

18. Harris WF. The symplecticity and relationships among the fundamental properties in linear optics. S Afr Optom 2010 69 3-13.

19. Harris WF. The log-transference and an average Gaussian eye. S Afr Optom 200564 84-88.

20. Harris WF and Cardoso JR. The exponential-mean-logtransference as a possible representation of the optical character of an average eye. Ophthal Physiol Opt 200626380 383.

21. Cardoso JR and Harris WF. Transformations of ray transferences of optical systems to augmented Hamiltonian matrices and the problem of the average eye. S Afr Optom $2007 \mathbf{6 6}$ 56-61.

22. Harris WF. Quantitative analysis of transformed ray transferences of optical systems in a space of augmented Hamiltonian matrices. S Afr Optom 200766 62-67.

23. Harris WF. Power vectors versus power matrices, and the mathematical nature of dioptric power. Optom Vis Sci 2007 84 1060-1063.

24. Gillan WDH. Variation in surface power and thickness of a moderately keratoconic cornea. S Afr Optom 2008 67 4-10.

25. Harris WF. Global and local contributions to corneal surface curvature: a simple model. S Afr Optom 200867 11-16.

26. Harris WF, Gillan WDH. Global and local contributions to curvature of a moderately keratoconic cornea. S Afr Optom 200867 17-20.

27. Rubin A, Mathebula SD. Global and local contributions to variation in surface curvature of healthy corneas. SAfr Optom [in preparation].

28. Harris WF. Optical axes of eyes and other optical systems. Optom Vis Sci 86 537-541. 